\title{
Experiment and Analysis on the Free Dynamics of a Shallow Arch After an Impact Load at the End
}

e-mail: jschen@ccms.ntu.edu.tw

Chun-Yi Liao

Graduate Student,

Department of Mechanical Engineering, National Taiwan University, Taipei, Taiwan 10617
In this paper we consider a sinusoidal arch with one end pinned in space while the other end attached to a mass and supported by a spring. The supporting wall of the spring is moved a distance quasi-statically to initiate preload in the arch and the spring. The assembly is then set in motion by an impact at the attached mass. The condition under which the arch may snap to the other side dynamically depends on the initial speed of the attached mass due to impact. Sufficient condition on the initial speed against dynamic snap-through is formulated based on the concept of minimum energy barrier. The effects of damping on the transient response of the assembly are also discussed. An experimental setup is designed to measure the transient response of the arch following the impact and the critical initial speed of the attached mass. The experimental results are in good agreement with theoretical predictions. [DOI: 10.1115/1.1827245]

\section{Introduction}

An arch subjected to lateral loads may become elastically unstable. If the initial height of the arch is of the same order as the span of the arch, the buckling deformation is nearly inextensional. On the other hand, an arch is termed shallow if the initial height is much smaller than the span. When the lateral load of a shallow arch reaches a critical value the deformed shape may undergo a sudden jump called snap-through buckling. The buckling deformation of a shallow arch will be extensional rather than inextensional. Depending on how the lateral load is applied, the snapthrough buckling of a shallow arch can be divided into two categories, i.e., static buckling and dynamic buckling. In the case of static buckling, the lateral load is applied in a quasi-static manner. The first theoretical prediction on the static critical load was conducted by Timoshenko in 1935 [1], in which a pinned sinusoidal arch was subjected to a uniformly distributed load. Fung and Kaplan [2] extended the research by considering a flexibly supported shallow arch under various kinds of lateral loading. Fung and Kaplan also conducted a series of experiments on the pin-ended arches having rigid simple supports. Gjelsvik and Bonder [3] presented a complete theoretical and experimental analysis on a clamped arch under a central concentrated load. Franciosi et al. [4] extended the conventional limit analysis to the collapse of arches under repeated loading. Schreyer and Masur [5] analyzed a clamped circular arch and demonstrated that the existence of a bifurcation of the equilibrium state is not an adequate condition for the use of the asymmetric buckling criterion. Lee and Murphy [6] considered the inelastic buckling of a clamped circular arch made of work-hardening material. Simitses [7] studied the effect of an elastic foundation on the critical loads of a sinusoidal arch. Roorda [8] conducted a series of experiments to study the effect of small imperfection on the buckling of elastic structures, including a laterally loaded circular arch.

In the case when the lateral load is applied suddenly instead of in quasi-static manner, the phenomenon is dynamic and much more complicated. Generally speaking, the methodologies used in estimating dynamic critical loads of elastic structures can be clas-

Contributed by the Applied Mechanics Division of THE AMERICAN SOCIETY OF MECHANICAL ENGINEERS for publication in the ASME JOURNAL OF APPLIED MECHANICS. Manuscript received by the Applied Mechanics Division, July 15, 2003; final revision, March 18, 2004. Associate Editor: N. Sri Namachchivaya. Discussion on the paper should be addressed to the Editor, Prof. Robert M. McMeeking, Journal of Applied Mechanics, Department of Mechanical and Environmental Engineering, University of California-Santa Barbara, Santa Barbara, CA 93106-5070, and will be accepted until four months after final publication in the paper itself in the ASME JOURNAL OF APPLIED MECHANICS. sified in two groups [9]. The first approach is to study the total energy and the phase plane of the system. By this method sufficient conditions for dynamic stability may be established. The first theoretical prediction of dynamic buckling load was conducted by Hoff and Bruce in 1954 [10], in which they studied the stability of a sinusoidal arch under unit step loading and ideal impulsive loading. Hsu [11,12] and Hsu et al. [13] studied the effects various parameters on the stability of a flexibly supported sinusoidal arch under impulsive and other types of time-varying loads. Xu et al. [14] considered a shallow arch elastically supported at both ends in the lateral direction and under impulsive loading. This approach provides a lower bound of the dynamic critical load.

The second approach is to solve the equations of motion numerically to obtain the system response and identify the critical load for specified system parameters. This approach provides a more accurate prediction of the critical load at the expense of large amount of calculation. Humphreys [15] performed both numerical and experimental studies on the dynamic snap-through of a circular arch under uniform impulsive loading. Lock [16] used a numerical integration method and an infinitesimal stability analysis to predict the dynamic critical load of a sinusoidal arch under a step loading. Huang and Nachbar [17] added the effects of geometric imperfection and viscoelastic behavior. Ariaratnam and Sankar [18] studied the dynamic buckling of a shallow arch under stochastic loads. Fulton and Barton [19] introduced a different criterion for dynamic stability. Sundararajan and Kumani [20] investigated the dynamic stability of a shallow arch under inclined loads. Lo and Masur [21] presented a hybrid method for snapthrough stability analysis, which incorporates an integral equation formulation in conjunction with a finite element method. Johnson and Mclvor [22] investigated numerically the effects of the spatial distribution of impulsive loads and damping [23] on the dynamic snap-through of a shallow arch. Huang and Plaut [24] studied the dynamic stability of a shallow arch under pulsating loads. Gregory and Plaut [25] and Donaldson and Plaut [26] discussed the stability boundaries for arches that are loaded by two independent sets of dynamic loads.

It is noted from the earlier literature review that while theoretical development on dynamic snap-through of shallow arches is quite well-established, experimental investigation [15] is relatively rare compared to the static case, partly because of the difficulty in applying prescribed time-varying lateral force. In this paper we will investigate, both theoretically and experimentally, a dynamic problem which involves an elastically supported arch subjected to impact at the end, as shown in Fig. 1. One end of the arch is pinned in space, while the other end is attached to a mass 


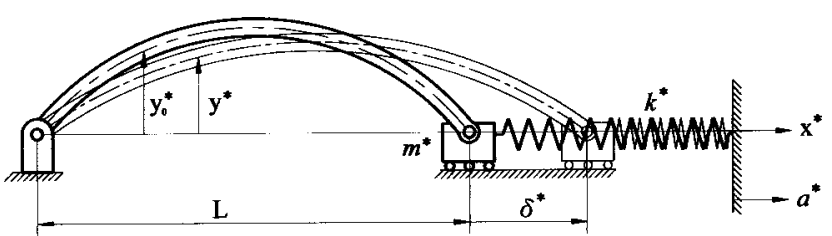

Fig. 1 Schematic diagram of a flexibly supported arch under impact at the end

and supported by a spring. The arch is not subjected to any lateral load. After a quasi-static movement of the supporting wall to preload the arch and the spring, the attached mass is subjected to an impact and attains a substantial initial speed. The arch-massspring assembly is then set in motion. The question we are interested in this paper is whether the arch will snap to the other side before it settles to a steady state position. Intuitively, if the impact is minor the arch will return to its original position after the vibration following impact is damped out. On the other hand, if the impact is more severe and the initial speed of the attached mass reaches a critical value, the arch may snap to the other side. Theoretical prediction of the critical initial speed will be presented and verified later by an experiment. By adjusting the stiffness of the supporting spring and the height of the arch, the assembly can be used as a mechanical warning device against severe impact.

\section{Equations of Motion}

Figure 1 shows a flexibly supported shallow arch, with one end pinned in space while the other end attached to a mass $m^{*}$ and supported by a spring with spring constant $k^{*}$. We assume that both the arch and the spring are in unstrained state initially. The initial shape of the arch when it is unstrained is $y_{0}^{*}\left(x^{*}\right)$ with the two ends being separated by a distance $L$. Before time $t^{*}=0$ we move quasi-statically the supporting wall of the spring a distance $a^{*}$ to the right. The arch-mass-spring system is then in a stable equilibrium position with the distance of the two ends of the arch being increased by an amount $\delta_{i}^{*}$, while the spring being stretched a distance $\delta_{i}^{*}-a^{*}$. The shape of the prestressed arch is denoted by $y_{i}^{*}\left(x^{*}\right)$. The axial thrust throughout the arch and the spring is $p_{i}^{*}$. If the supporting wall of the spring is moved to the left then $a^{*}$ is negative. We assume that at time $t^{*}=0$ the attached mass of the prestressed assembly is under impact by an object and attains an initial speed $\dot{\delta}^{*}(0)=v_{i}^{*}$. The arch-massspring system is then set in motion with the shape of the vibrating arch being denoted by $y^{*}\left(x^{*}, t^{*}\right)$. The equation of motion of the arch can be written as

$$
\rho A y_{, t^{*} t^{*}}^{*}=-E I\left(y^{*}-y_{0}^{*}\right)_{, x^{*} x_{x} * x^{*}}+\left(p^{*}-m^{*} \ddot{\delta}^{*}\right) y_{, x^{*} x^{*}}^{*}
$$

The parameters $E, \rho, A$, and $I$ are Young's modulus, mass density, area, and area moment of inertia of the cross section of the arch. The comma represents partial differentiation, while the overhead dot represents the derivative with respect to time. In writing Eq. (1) we assume that the curvature of the arch is small and can be approximated by $-\left(y^{*}-y_{0}^{*}\right)_{, x^{*} x^{*}}$. An arch is termed high arch when its curvature cannot be treated as small. We also assume that the effects of rotary inertia and shearing deformations are neglected. Strains are assumed to remain within the elastic limit and Hooke's law is valid. The term $p^{*}-m^{*} \ddot{\delta}^{*}$ is the axial thrust in the arch, while $p^{*}$ alone is the axial force in the spring

$$
p^{*}=-k^{*}\left(\delta^{*}-a^{*}\right)
$$

We assume that the axial thrust is constant along the arch and is thus a function only of the time. The moving distance $\delta^{*}$ of the attached mass from its initial rest position before the quasi-static wall movement can be related to the arch shape $y^{*}\left(x^{*}, t^{*}\right)$ by considering the equilibrium of the attached mass as

$$
\delta^{*}=\frac{\left(p^{*}-m^{*} \ddot{\delta}^{*}\right) L}{E A}+\frac{1}{2} \int_{0}^{L}\left[\left(y_{0, x^{*}}^{*}\right)^{2}-\left(y_{, x^{*}}^{*}\right)^{2}\right] d x^{*}
$$

The first term on the right-hand side of Eq. (3) represents the effect of extensibility of the arch, while the second term represents the effect of shape change (mainly due to rotation). From Eqs. (2) and (3) we can derive $p^{*}$ as

$$
\begin{aligned}
p^{*}(t)= & \frac{k^{*}}{E A+k^{*} L}\left\{L m^{*} \ddot{\delta}^{*}+E A a^{*}-\frac{E A}{2} \int_{0}^{L}\left[\left(y_{0, x *}^{*}\right)^{2}\right.\right. \\
& \left.\left.-\left(y_{, x^{*}}^{*}\right)^{2}\right] d x^{*}\right\}
\end{aligned}
$$

The parameters $\delta_{i}^{*}, p_{i}^{*}$, and function $y_{i}^{*}\left(x^{*}\right)$, which refer to the mass position, axial force, and deformed shape of the arch before impact, can be calculated from Eqs. (1), (3), and (4) by ignoring all the terms involving differentiation with respect to time.

Equations (1) and (4) can be nondimensionalized to the forms

$$
\begin{aligned}
& y_{, t t}=-\left(y-y_{0}\right)_{, x x x x}+(p-m \ddot{\delta}) y_{, x x} \\
& p=\frac{k}{2 \pi} \int_{0}^{\pi}\left(y_{, x}^{2}-y_{0, x}^{2}\right) d x+a+k m \ddot{\delta}
\end{aligned}
$$

where

$$
\begin{gathered}
y=\frac{y^{*}}{r}, \quad y_{0}=\frac{y_{0}^{*}}{r}, \quad x=\frac{\pi x^{*}}{L}, \quad t=\frac{\pi^{2} t^{*}}{L^{2}} \sqrt{\frac{E I}{A \rho}}, \\
p=\frac{p^{*} L^{2}}{\pi^{2} E I}, \quad k=\frac{k^{*}}{k^{*}+E A / L} \\
\delta=\frac{L \delta^{*}}{\pi^{2} r^{2}(1-k)}, \quad a=\frac{k L a^{*}}{\pi^{2} r^{2}}, \quad m=\frac{(1-k) I \pi^{4} m^{*}}{L^{3} A^{2} \rho}, \\
v_{i}=\frac{L^{3} v_{i}^{*}}{\pi^{4} r^{3}(1-k)} \sqrt{\frac{\rho}{E}}
\end{gathered}
$$

As a general rule in this paper, a variable without asterisk is a dimensionless counterpart of the one with asterisk. $r$ is the radius of gyration of the cross section of the arch. $p=1$ corresponds to the Euler buckling load for a perfectly straight simply supported beam. The dimensionless spring constant $k$ ranges from $0\left(k^{*}\right.$ $=0)$ to $1\left(k^{*} \rightarrow \infty\right)$. The boundary conditions for $y$ at $x=0$ and $\pi$ are

$$
\begin{aligned}
y(0)-y_{0}(0) & =y_{, x x}(0)-y_{0, x x}(0)=y(\pi)-y_{0}(\pi) \\
& =y_{, x x}(\pi)-y_{0, x x}(\pi)=0
\end{aligned}
$$

The initial shape of the unstrained arch is assumed to be in the form

$$
y_{0}(x)=h \sin x
$$

$h$ is the initial height of the arch. It is assumed that the shape of the arch can be expanded as

$$
y(x, t)=y_{0}+\sum_{n=1}^{\infty} \alpha_{n}(t) \sin n x
$$

After substituting Eqs. (8) and (9) into (5) and (6) we obtain the equations governing the generalized coordinates $\alpha_{n}$ :

$$
\begin{gathered}
\ddot{\alpha}_{1}=-\alpha_{1}-(p-m \ddot{\delta})\left(h+\alpha_{1}\right) \\
\ddot{\alpha}_{n}=-n^{4} \alpha_{n}-n^{2}(p-m \ddot{\delta}) \alpha_{n} \quad n=2,3, \ldots
\end{gathered}
$$

where

$$
p=k\left(\frac{h}{2} \alpha_{1}+\frac{1}{4} \sum_{i=1}^{\infty} i^{2} \alpha_{i}^{2}\right)+a+k m \ddot{\delta}
$$


It is noted that the parameter $\ddot{\delta}$ in Eqs. (10), (11), and (12) is still unknown. To obtain the additional equation of motion accounting for $\delta$ we substitute Eq. (4) into Eq. (3), nondimensionalize, and discretize by using Eq. (9):

$$
m \ddot{\delta}=-\delta-\left(\frac{h}{2} \alpha_{1}+\frac{1}{4} \sum_{i=1}^{\infty} i^{2} \alpha_{i}^{2}\right)+\frac{a}{1-k}
$$

Equations (10)-(13) are the discretized equations of motion of the assembly.

\section{Equilibrium Configurations}

We first study the equilibrium configurations of the prestressed arch-mass-spring assembly following the quasi-static wall movement $a$. The shape of the prestressed arch can be determined from Eqs. (10) and (11) by neglecting all the acceleration terms. There are two different types of equilibrium configurations, i.e., onemode and two-mode solutions. It can be easily shown that Eqs. (10) and (11) do not admit an equilibrium configuration with more than two modes.

One-Mode Solution $y=y_{0}+\alpha_{1} \sin x$.

$\alpha_{1}$ satisfies the following cubic equation

$$
a\left(\alpha_{1}+h\right)=-\frac{\alpha_{1}}{4}\left(k \alpha_{1}^{2}+3 k h \alpha_{1}+2 k h^{2}+4\right)
$$

After defining parameter $a_{1}$ as

$$
a_{1}=\frac{k h^{2}}{4}-\frac{3}{4}\left(4 k h^{2}\right)^{1 / 3}-1
$$

we can make the following observations.

1. If $a>a_{1}$, then there is only one equilibrium configuration denoted by $P_{0}$.

2. If $a<a_{1}$, then there are three equilibrium configurations $P_{0}, P_{1}^{+}$, and $P_{1}^{-}$, where

$$
\alpha_{1}\left(P_{1}^{-}\right)<-h-\left(\frac{2 h}{k}\right)^{1 / 3}<\alpha_{1}\left(P_{1}^{+}\right)<-h<\alpha_{1}\left(P_{0}\right)
$$

3. If $a=a_{1}$, then the configurations $P_{1}^{+}$and $P_{1}^{-}$coincide. We denote this special one-mode configuration as $P_{1}$. Also

$$
\alpha_{1}\left(P_{1}\right)=-h-\left(\frac{2 h}{k}\right)^{1 / 3}
$$

Two-Mode Solution $y=y_{0}+\alpha_{1} \sin x+\alpha_{j} \sin j x$.

It can be shown that a two-mode solution always contains the first mode $\alpha_{1} \sin x$. For this case the solutions can be written explicitly

$$
\begin{gathered}
\alpha_{1}=\frac{-j^{2} h}{j^{2}-1} \\
\alpha_{j}= \pm \frac{2}{j} \sqrt{\frac{a_{j}-a}{k}}
\end{gathered}
$$

where

$$
a_{j}=\frac{\left(j^{2}-2\right) j^{2} h^{2} k}{4\left(j^{2}-1\right)^{2}}-j^{2}
$$

These configurations are denoted by $P_{1 j}^{+}$and $P_{1 j}^{-}$, which exist only when

$$
a<a_{j}
$$

It is noted that in both Eqs. (15) (for $a_{1}$ ) and (20) (for $a_{j}$ ) the parameters $k$ and $h$ appear together in the form $k h^{2}$. By comparing Eqs. (15) and (20) we observe that

$$
a_{1} \geqslant a_{j}
$$

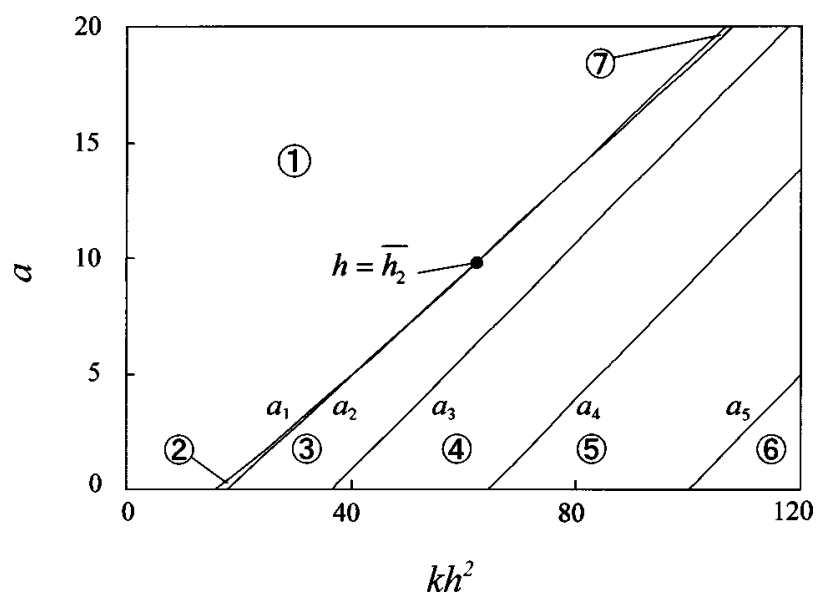

Fig. $2 a_{j}$ curves on the $k h^{2}-a$ plane

The special initial height which renders $a_{1}=a_{j}$ is denoted by $\bar{h}_{j}$, where

$$
k \bar{h}_{j}^{2}=2\left(j^{2}-1\right)^{3} \quad j=2,3,4, \ldots
$$

The values of some of $k \bar{h}_{j}^{2}$ are $k \bar{h}_{2}^{2}=54, k \bar{h}_{3}^{2}=1024$, etc. Figure 2 shows the $a_{j}$ curves in the $k h^{2}-a$ plane. These $a_{j}$ curves divide the $k h^{2}-a$ plane into several regions. The black dot at $h=\bar{h}_{2}$ signifies the touching point between $a_{1}$ and $a_{2}$ curves. For a given set of $k h^{2}$ and $a$, we can determine the number of equilibrium configurations. For instance, if the point $\left(k h^{2}, a\right)$ fall in the region 1 , then there is only one equilibrium configuration $P_{0}$. For the parameter ranges in Fig. 2 there are seven regions, whose equilibrium configurations are listed in Table 1.

\section{Stability of Equilibrium Configurations}

First of all, the dimensionless total energy $H$ of any configuration can be calculated as

$$
\begin{aligned}
H= & \frac{2}{\pi} \int_{0}^{\pi}\left[\left(y_{, t}\right)^{2}+\left(y_{, x x}-y_{0, x x}\right)^{2}\right] d x+2(p-m \ddot{\delta})^{2}+\frac{2 p^{2}(1-k)}{k} \\
& +2 m \dot{\delta}^{2}(1-k)
\end{aligned}
$$

The two terms in the integral represent the kinetic energy and the bending strain energy. The second term is the strain energy due to the axial force. The third term is the strain energy of the spring. The last term is the kinetic energy of mass. For an equilibrium configuration corresponding to a specified wall movement $a$, the kinetic energy is zero and the total energy consists of only the strain energy $U$ :

$$
U=\frac{2}{\pi} \int_{0}^{\pi}\left(y_{, x x}-y_{0, x x}\right)^{2} d x+\frac{2 p^{2}}{k}
$$

Table 1 Equilibrium configurations in various regions of Fig. 2

\begin{tabular}{lll}
\hline \hline Region & \multicolumn{1}{c}{ Equilibrium configuration } & Stability of $P_{1}^{-}$ \\
\hline 1 & $P_{0}$ & Stable \\
2 & $P_{0}, P_{1}^{ \pm}$ & Stable \\
3 & $P_{0}, P_{1}^{ \pm}, P_{12}^{ \pm}$ & Stable \\
4 & $P_{0}, P_{1}^{ \pm}, P_{12}^{ \pm}, P_{13}^{ \pm}$ & Stable \\
5 & $P_{0}, P_{1}^{ \pm}, P_{12}^{ \pm}, P_{13}^{ \pm}, P_{14}^{ \pm}$ & Stable \\
6 & $P_{0}, P_{1}^{ \pm}, P_{12}^{ \pm}, P_{13}^{ \pm}, P_{14}^{ \pm}, P_{15}^{ \pm}$ & Unstable \\
7 & $P_{0}, P_{1}^{ \pm}$ & \\
\hline \hline
\end{tabular}


For the two-mode configurations $P_{1 j}^{+}$and $P_{1 j}^{-}$the strain energy are equal, and can be written as

$$
U\left(P_{1 j}^{+}\right)=U\left(P_{1 j}^{-}\right)=\frac{1}{k}\left(\frac{j^{4} k h^{2}}{j^{2}-1}-2 j^{4}-4 j^{2} a\right)
$$

For the one-mode solutions the strain energy is

$$
U=\alpha_{1}^{2}+\frac{2 p^{2}}{k}
$$

where $\alpha_{1}$ and $p$ are the generalized coordinate and the axial thrust of the one-mode solution. The physical total energy $H^{*}$ and strain energy $U^{*}$ are related to $H$ and $U$ by

$$
H=\frac{4 A L^{3} H^{*}}{\pi^{2} E I^{2}}, \quad U=\frac{4 A L^{3} U^{*}}{\pi^{2} E I^{2}}
$$

In order to study the stability of the equilibrium configuration with shape $\bar{y}$, we perturb the equilibrium shape by a small amount $\varepsilon \hat{y}$ to examine how the strain energy changes. $\varepsilon$ is a small positive number. The strain energy $U$ of the perturbed configuration can be expanded in terms of $\varepsilon$ as

$$
\begin{aligned}
U(\bar{y}+\varepsilon \hat{y})-U(\bar{y})= & \varepsilon\left\{\frac{4}{\pi} \int_{0}^{\pi}\left[\left(\bar{y}_{, x x}-y_{0, x x}\right) \hat{y}_{, x x}+\overline{p y}_{, x} \hat{y}_{, x}\right] d x\right\} \\
& +\varepsilon^{2}\left\{\frac{2}{\pi} \int_{0}^{\pi}\left[\left(\hat{y}_{, x x}\right)^{2}+\bar{p}\left(\hat{y}_{, x}\right)^{2}\right] d x\right. \\
& \left.+\frac{2 k}{\pi^{2}}\left[\int_{0}^{\pi} \bar{y}_{, x} \hat{y}_{, x} d x\right]^{2}\right\} \\
& +\varepsilon^{3}\left\{\frac{2 k}{\pi^{2}}\left[\int_{0}^{\pi} \bar{y}_{, x} \hat{y}_{, x} d x\right]\left[\int_{0}^{\pi}\left(\hat{y}_{, x}\right)^{2} d x\right]\right\} \\
& +\varepsilon^{4}\left\{\frac{k}{2 \pi^{2}}\left[\int_{0}^{\pi}\left(\hat{y}_{, x}\right)^{2} d x\right]^{2}\right\}
\end{aligned}
$$

$\bar{p}$ is the axial force of the equilibrium configuration $\bar{y}$, and can be calculated from Eq. (10) as

$$
\bar{p}=\frac{-\alpha_{1}}{\alpha_{1}+h}
$$

To prove that an equilibrium shape $\bar{y}$ is stable we have to show that the energy difference $U(\bar{y}+\varepsilon \hat{y})-U(\bar{y})$ is positive for any $\hat{y} \neq 0$. On the other hand, to prove that $\bar{y}$ is an unstable equilibrium shape, we only need to find one $\hat{y} \neq 0$ which renders $U(\bar{y}+\varepsilon \hat{y})$ $-U(\bar{y})$ negative. After integrating by parts and using the fact that $\bar{y}$ satisfies the static equilibrium equations it can be shown that the coefficient of $\varepsilon$ in Eq. (29) is zero. To determine the stability we next examine the second variation of the strain energy

$$
\delta^{2} U=\frac{2}{\pi} \int_{0}^{\pi}\left[\left(\hat{y}_{, x x}\right)^{2}+\bar{p}\left(\hat{y}_{, x}\right)^{2}\right] d x+\frac{2 k}{\pi^{2}}\left[\int_{0}^{\pi} \bar{y}_{, x} \hat{y}_{, x} d x\right]^{2}
$$

In some cases $\delta^{2} U$ is zero identically, and higher order variation is needed. More details of the above energy method can be found in Ref. [27], and the conclusions are summarized in the following.

One-Mode Solutions: $P_{0}$ is always stable. $P_{1}^{+}$is always unstable. If $h \leqslant \overline{h_{2}}$, then $P_{1}^{-}$is stable if and only if $a<a_{1}$. On the other hand, if $h>\overline{h_{2}}$, then $P_{1}^{-}$is stable if and only if $a<a_{2}$.

Two-Mode Solutions: $P_{1 j}^{+}$and $P_{1 j}^{-}$are always unstable.

From the earlier analysis, we know that for any combination of $a$ and $k h^{2}$ there are at most two stable configurations. One of them is always $P_{0}$, and the other possible stable configuration is $P_{1}^{-}$. In Table 1 we also identify the regions with stable $P_{1}^{-}$. For those quasi-static wall movements $a$, which result in only one stable equilibrium position $P_{0}$, the assembly will return to the original position following impact. On the other hand, for those wall movements which render the existence of two stable positions $P_{0}$ and $P_{1}^{-}$, then it is possible for the arch to snap and settle to $P_{1}^{-}$ following the impact. It is the purpose of this paper to determine the lowest possible initial speed of the impacted mass, below which the arch-mass-spring assembly is in no danger to snap. This lowest initial speed is called the critical initial speed.

\section{Snap-Through Criterion}

While it is in general difficult to determine the necessary and sufficient condition for dynamic snap-through to occur, we can establish the sufficient conditions against dynamic snap-through in terms of the dimensionless total energy $H$ of the vibrating archmass-spring assembly following the impact. The basic idea of the snap-through criterion is that if the total energy gained by the assembly from the impact is smaller than the minimum energy barrier lying between the nearest stable equilibrium position and the distant stable one, then the arch has no chance to snap dynamically. The energy barrier can be proved to be the strain energy of either the unstable configuration $P_{1}^{+}$or $P_{12}^{ \pm}$, depending on the parameters $k, h$ and $a$ [27]. The sufficient conditions against dynamic snap-through from $P_{0}$ to $P_{1}^{-}$can then be stated in the following.

Case (1) $h \leqslant \overline{h_{2}}$ and $a_{2} \leqslant a<a_{1}$ : The sufficient condition against snap-through is $H_{i}<U\left(P_{1}^{+}\right)$, where $H_{i}$ is the total energy immediately following impact.

Case (2) $a<a_{2}$ : The sufficient condition against snap-through is $H_{i}<U\left(P_{12}^{ \pm}\right)$.

Figures 3(a), 3(b), and 3(c) show the equilibrium positions and strain energy contours for three typical situations. In Fig. 3(a) $k$ $=0.4, h=10$, and $a=3$, which falls in region 3 of Fig. 2 . There are five equilibrium positions in this region, among them $P_{12}^{ \pm}$are the saddle points whose strain energy is the energy barrier preventing the system from snapping from position $P_{0}$ to another stable position $P_{1}^{-}$. In Fig. 3(b) $k=0.2, h=10$, and $a=0.6$, which falls in region 2 of Fig. 2. There are three equilibrium positions in this region, among them $P_{1}^{+}$is a saddle point whose strain energy serves as the energy barrier. In Fig. 3(c) $k=0.8, h=10$, and $a$ $=13.8$, which falls in region 7 of Fig. 2 . There are three equilibrium positions in this region, among them $P_{0}$ is the only stable equilibrium position. $P_{1}^{-}$becomes a saddle point in this case.

\section{Effect of Damping}

Figures $4(a), 4(b)$, and $4(c)$ show the deformation history of an arch with $h=10, k=0.4, a=3$, and $m=0.001$, which falls in region 3 of Fig. 2. In calculating the response we modify Eq. (10) by adding a damping parameter $\mu$ :

$$
\ddot{\alpha}_{1}=-\mu \dot{\alpha}_{1}-\alpha_{1}-(p-m \ddot{\delta})\left(h+\alpha_{1}\right)
$$

The relation between $\mu$ and its physical counterpart $\mu^{*}$ is

$$
\mu=\frac{\mu^{*} L^{2}}{\pi^{2} \rho A r} \sqrt{\frac{\rho}{E}}
$$

The initial speed $v_{i}$ is set to be 110 . The damping $\mu$ used in Figs. $4(a), 4(b)$, and $4(c)$ are $0.2,0.3$, and 0.6 , respectively. The initial conditions are

$$
\begin{gathered}
\alpha_{i}(0)=0, \quad i=1,2,3, \ldots \\
\dot{\alpha}_{1}(0)=-\frac{2 v_{i}}{h}, \\
\dot{\alpha}_{i}(0)=0, \quad i=2,3,4, \ldots
\end{gathered}
$$

For the small damping case in Fig. 4(a) the energy gained by the assembly is large enough to surpass the energy barrier $U\left(P_{12}^{ \pm}\right)$and 
(a)

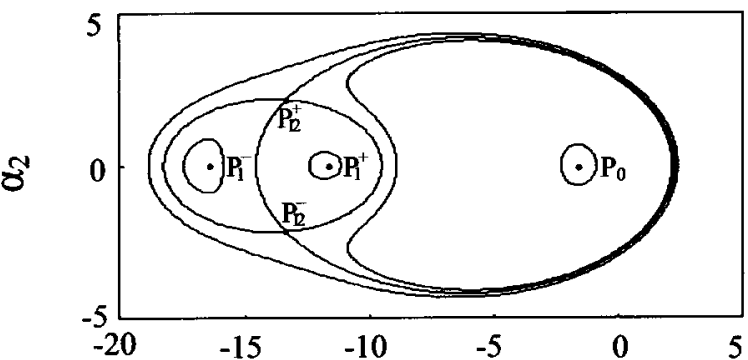

(b)

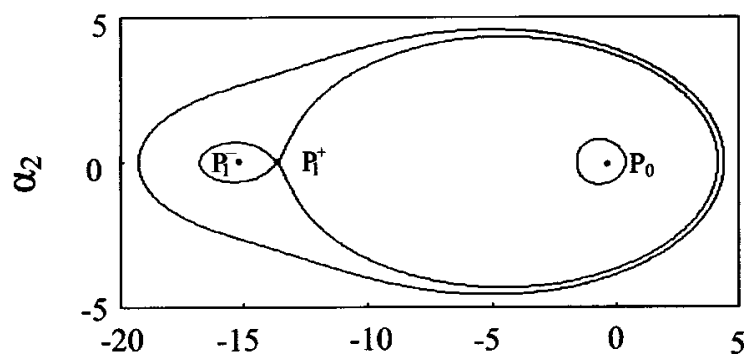

(c)

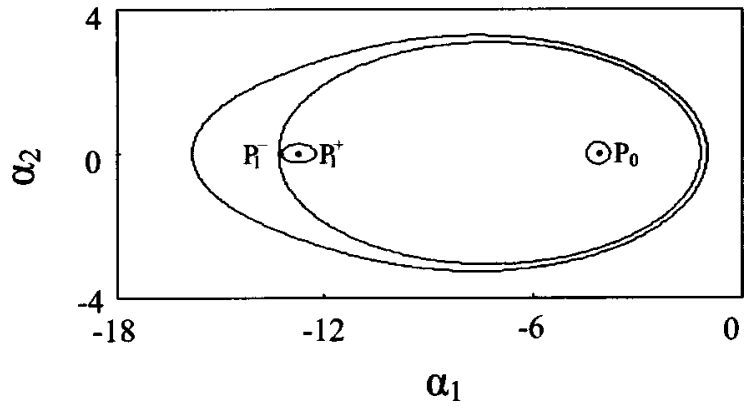

Fig. 3 Strain energy contours for (a) $k=0.4, h=10, a=3$, (b) $k=0.2, h=10, a=0.6$, (c) $k=0.8, h=10, a=13.8$

$\alpha_{1}$ can reach $\alpha_{1}\left(P_{1}^{-}\right)$. However, due to small damping, the arch is snapped back and finally settles to position $P_{0}$. For the medium damping case in Fig. 4(b), the assembly not only gains enough energy to surpass $U\left(P_{12}^{ \pm}\right)$and $\alpha_{1}$ can reach $\alpha_{1}\left(P_{1}^{-}\right)$, the damping also prevents it from snapping back to $P_{0}$. The arch settles to $P_{1}^{-}$ eventually. In Fig. 4(c) the damping is so large that it prevents the arch from surpassing $U\left(P_{12}^{ \pm}\right)$, and the arch has no choice but to settle to $P_{0}$. The existent equilibrium positions are plotted as dashed horizontal lines for reference. These examples demonstrate that damping plays an important role in dynamic snap-through when the total energy of the assembly gained from impact is greater than the energy barrier.

\section{Critical Initial Speed}

Another important factor in determining whether snap-through will occur is the end initial speed. Apparently, for very small initial speed no snap-through is possible. As $v_{i}$ increases, on the other hand, snap-through might occur. It is therefore possible to define a critical initial speed $v_{\text {cr }}$ below which no snap-through is possible even if there exist two stable equilibrium positions. From the snap-through criterion discussed previously, we can derive the expression of the critical initial speed as follows.

For the case when $h \leqslant \overline{h_{2}}$ and $a_{2} \leqslant a<a_{1}$ the energy barrier is $U\left(P_{1}^{+}\right)$. From the condition $H_{i}\left(v_{c r}\right)=U\left(P_{1}^{+}\right)$, where $H_{i}\left(v_{c r}\right)$ is the total energy of the system immediately after the attached mass attains initial speed $v_{\mathrm{cr}}$ from the impact, it can be found that the critical initial speed $v_{\text {cr }}$ for this case is (a)

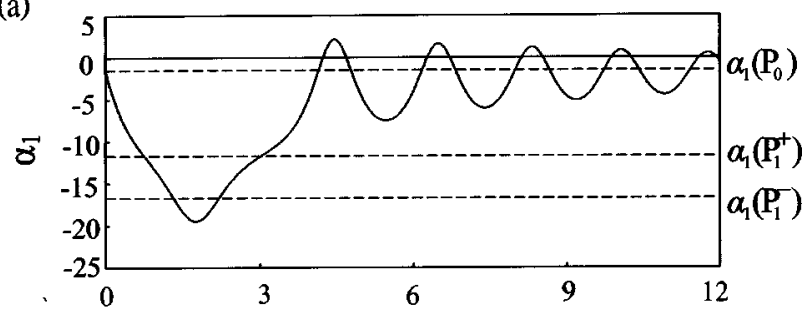

(b)
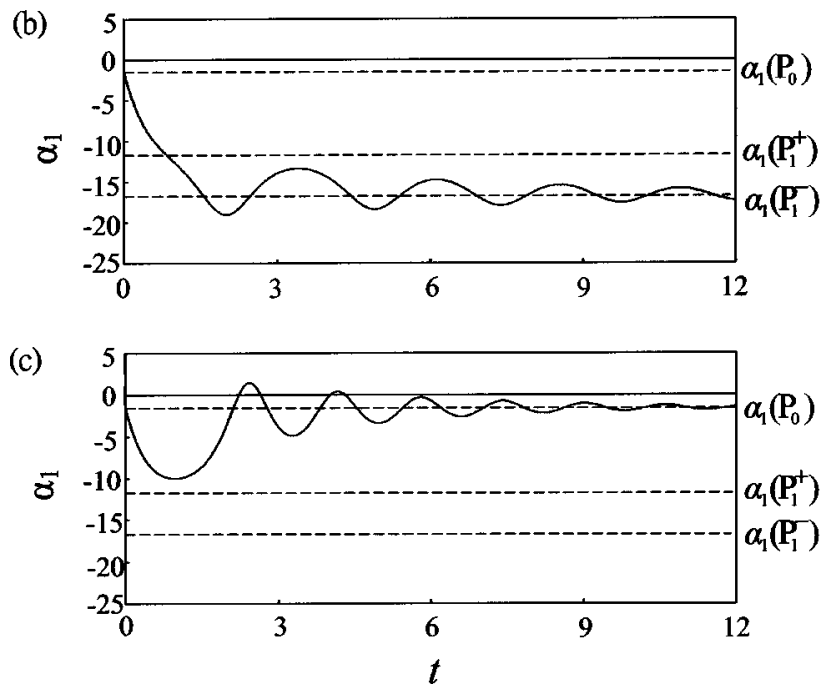

Fig. 4 Effect of damping $\mu$ on the response of the assembly with $h=10, k=0.4, a=3, v_{i}=110, m=0.001$. (a) $\mu=0.2$, (b) $\mu=0.3$, (c) $\mu=0.6$.

$$
v_{\mathrm{cr}}=\frac{h\left[k \alpha_{1}^{2}\left(P_{1}^{+}\right)-k \alpha_{1}^{2}\left(P_{0}\right)+2 p^{2}\left(P_{1}^{+}\right)-2 p^{2}\left(P_{0}\right)\right]^{1 / 2}}{\left[2 m h^{2} k(1-k)+4 k\right]^{1 / 2}}
$$

$p\left(P_{0}\right)$ and $p\left(P_{1}^{+}\right)$are the axial thrust of the assembly in the equilibrium positions $P_{0}$ and $P_{1}^{+}$, respectively. For the case when $a<a_{2}$ the energy barrier is $U\left(P_{12}^{ \pm}\right)$. From the condition $H_{i}\left(v_{\mathrm{cr}}\right)$ $=U\left(P_{12}^{ \pm}\right)$it can be found that the critical initial speed $v_{\mathrm{cr}}$ for this case is

$$
v_{\mathrm{cr}}=\frac{h\left[16 k h^{2}-96-48 a-3 k \alpha_{1}^{2}\left(P_{0}\right)-6 p^{2}\left(P_{0}\right)\right]^{1 / 2}}{\left[6 m h^{2} k(1-k)+12 k\right]^{1 / 2}}
$$

It is noted that the one-mode solution $P_{0}$ and $P_{1}^{+}$cannot be solved explicitly when $a \neq 0$. Therefore, the parameters $\alpha_{1}\left(P_{0}\right)$, $\alpha_{1}\left(P_{1}^{+}\right), p\left(P_{0}\right)$, and $p\left(P_{1}^{+}\right)$in Eqs. (35) and (36) can only be calculated numerically. On the other hand when $a=0$, i.e., the assembly is unstrained before impact, Eqs. (35) and (36) can be expressed in the following closed forms. For the case $18>k h^{2}>16$ :

$$
\begin{gathered}
v_{\mathrm{cr}}=\frac{h^{2}(3-c)}{4(1-c)}\left\{\frac{16+2 k h^{2}(1-c)^{2}}{k h^{2}\left[m h^{2}(1-k)+2\right]}\right\}^{1 / 2}, \\
\text { where } c=\sqrt{1-\frac{16}{k h^{2}}}
\end{gathered}
$$

For the case $k h^{2}>18$ :

$$
v_{\mathrm{cr}}=\frac{h}{2}\left[\frac{32 h^{2} k-192}{3 m h^{2} k(1-k)+6 k}\right]^{1 / 2}
$$




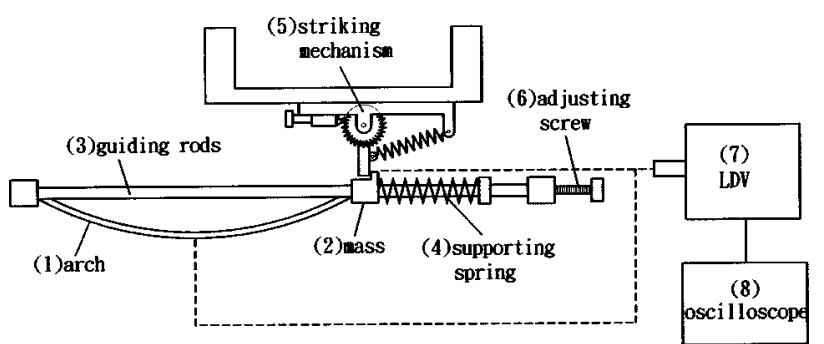

Fig. 5 Schematic diagram of the experimental setup

\section{Experimental Setup}

An experimental setup is designed to verify the earlier theoretical prediction. The schematic diagram of the setup is shown in Fig. 5. The function of each component in the setup is explained as follows. (1) The arch is made of aluminum strip with Young's modulus $70 \mathrm{GPa}$ and mass density $2800 \mathrm{~kg} / \mathrm{m}^{3}$. The length $L$ of the arch is $40 \mathrm{~cm}$ and the cross section is $25 \mathrm{~mm} \times 1.5 \mathrm{~mm}$. (2) Attached mass, which consists of a roller bearing and a linear bearing at the end, is scaled at $m^{*}=473 \mathrm{~g}$. The roller bearing is installed to simulate the pinned condition, while the linear bearing is to reduce the friction when the mass slides on the guiding rods (3). (4) Spring to support the arch in the axial direction, whose spring constant can be adjusted in the range $k^{*}=200-400 \mathrm{~N} / \mathrm{cm}$ by changing its working length. (5) A lock-and-release striking mechanism, which consists of a striking hammer and a spring to store the striking power. (6) An adjustable screw mechanism to control the quasi-static wall movement $a^{*}$. (7) An LDV system made by Polytec Co. (optical measurement head OFV-508 and electronic signal processor OFV-2802) to measure the speed of the sliding mass in the axial direction or the lateral speed at the midpoint (one at a time) of the arch. The converting ratio of the LDV signal is $1 \mathrm{~V}$ to $125 \mathrm{~mm} / \mathrm{s}$. The instrument can record a signal up to $15 \mathrm{~V}$. Displacement can be obtained by integrating the speed signal. (8) A digital oscilloscope is used to visually monitor and record the signals from the LDV system. A photograph of the laboratory setup is shown in Fig. 6.

\section{Transient Response Measurements}

Figure 7 shows the measured speed history of the attached mass following the impact. The parameters of the assembly are initial height $h^{*}=2.80 \mathrm{~cm}(h=65)$, spring constant $k^{*}=327 \mathrm{~N} / \mathrm{cm}(k$ $=0.0050)$, wall movement $a^{*}=0$. The complicated impact phenomenon between the striking hammer and the metallic flange on the sliding mass is not of our interest. Instead, we focus our attention to the initial speed gained by the sliding mass after the

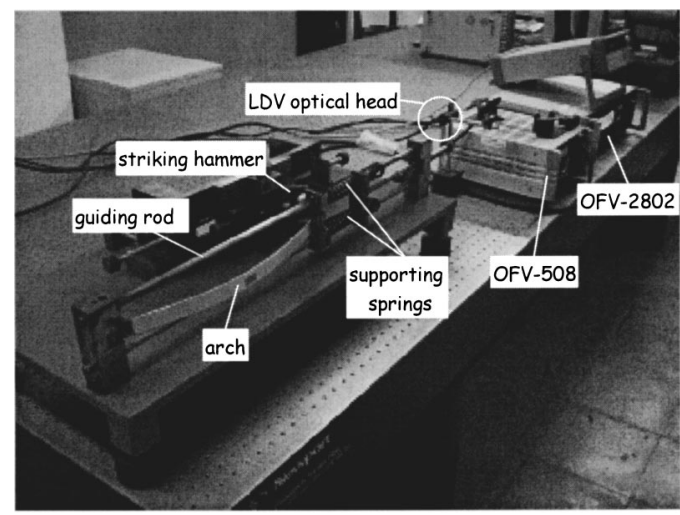

Fig. 6 A photograph of the experimental setup in the laboratory

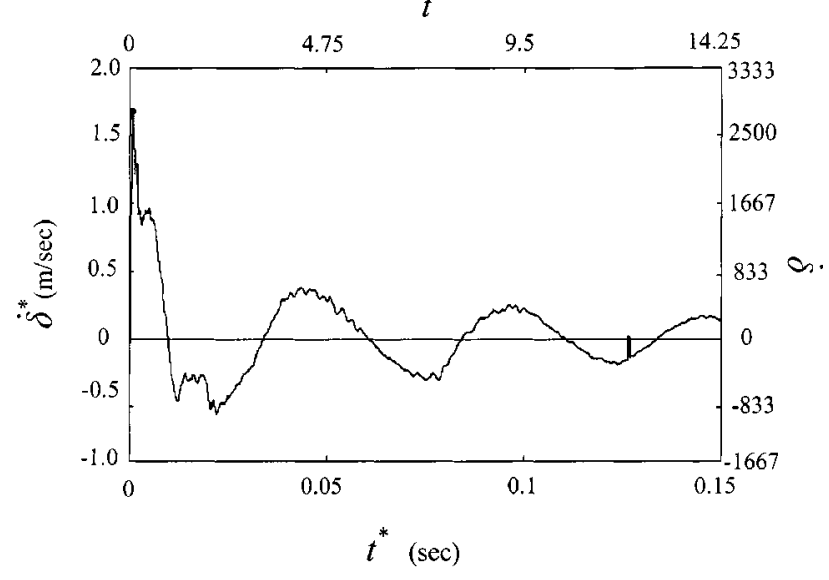

Fig. 7 Measured speed history of the attached mass following the impact. Parameters of the assembly are $h^{*}=2.80 \mathrm{~cm}$, $k^{*}=327 \mathrm{~N} / \mathrm{cm}, a^{*}=0$.

impact. The initial speed is measured at $v_{i}^{*}=1.72 \mathrm{~m} / \mathrm{s}\left(v_{i}\right.$ $=2860$ ) and is signified by a black dot in the figure. The rise time from impact to the speed peak is about $0.0005 \mathrm{~s}$. In this experiment snap-through occurs with the arch passing through the horizontal position at $0.015 \mathrm{~s}$ (the first crossing of the $\dot{\delta}^{*}=0$ line). For easy reference we present the results with both physical parameters (with asterisk) and the dimensionless ones (without asterisk). The same labeling style is adopted in the following figures.

The solid lines in Fig. 8 represent the measured lateral deflection history at the midpoint of the arch following the impact. The parameters of the assembly in Fig. $8(a)$ are $h^{*}=3.46 \mathrm{~cm}(h$ $=80), \quad k^{*}=206 \mathrm{~N} / \mathrm{cm}(k=0.0034), \quad v_{i}^{*}=1.44 \mathrm{~m} / \mathrm{s}\left(v_{i}=2400\right)$.

(a)
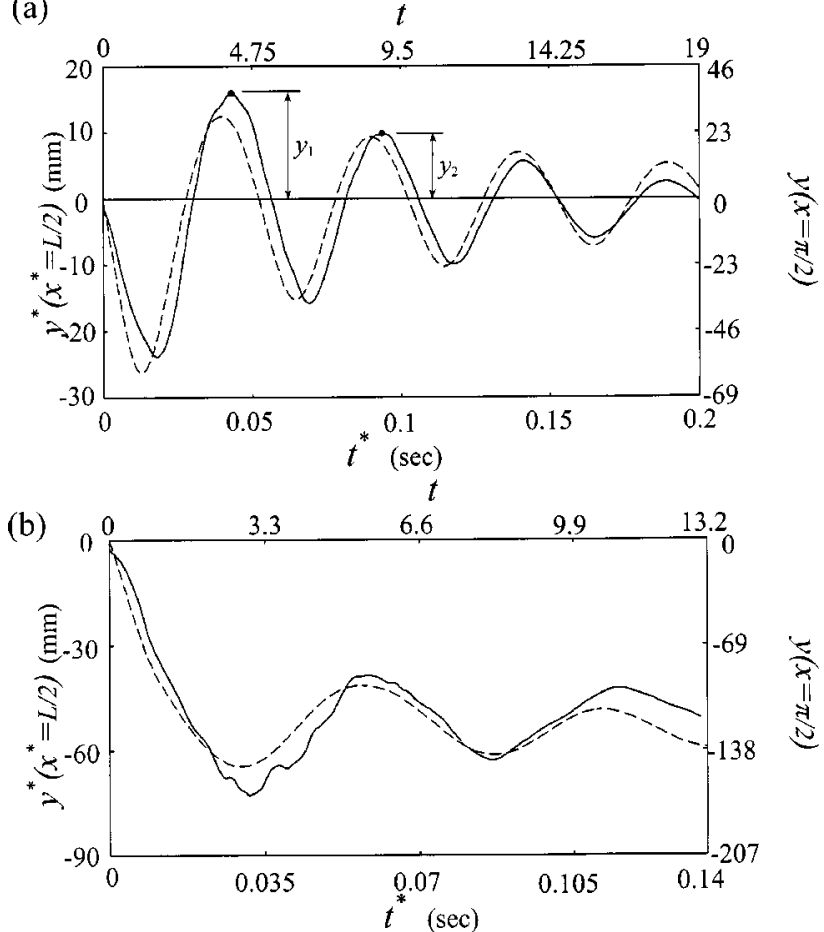

Fig. 8 Deflection history at the midpoint of the arch following the impact. The solid lines are the measured response while the dashed lines are the numerical results. (a) $h^{*}=3.46 \mathrm{~cm}$, $k^{*}=206 \mathrm{~N} / \mathrm{cm}, v_{i}^{*}=1.44 \mathrm{~m} / \mathrm{s}$. (b) $h^{*}=3.00 \mathrm{~cm}, k^{*}=333 \mathrm{~N} / \mathrm{cm}$, $v_{i}^{*}=1.79 \mathrm{~m} / \mathrm{s}$. 
No snap-through occurs in this case and the arch settles to the original shape eventually. Figure $8(b)$ shows the deflection history of another assembly with parameters $h^{*}=3.00 \mathrm{~cm}(h=70), k^{*}$ $=333 \mathrm{~N} / \mathrm{cm}(k=0.0051), \quad v_{i}^{*}=1.79 \mathrm{~m} / \mathrm{s}\left(v_{i}=2975\right) . \quad$ Snapthrough occurs in Fig. 8(b) and the arch settles eventually to the other side.

To simulate the motion of the assembly numerically, we have to estimate the damping of the system. The dissipating mechanism of the system comes from the friction in the moving parts and the material damping in the arch and the spring. We assume that the first mode is dominant in the dynamic response in Fig. 8(a). The damping factor may be estimated from the decaying rate of the two peaks as signified by black dots. The heights of the two peaks are measured at $y_{1}=38$ and $y_{2}=24$. The ratio of the damping of the system $\mu$ in Eq. (32) to a critical damping $\mu_{c}$ is [28]

$$
\frac{\mu}{\mu_{c}}=\frac{\ln \left(y_{1} / y_{2}\right)}{\left\{(2 \pi)^{2}+\left[\ln \left(y_{1} / y_{2}\right)\right]^{2}\right\}^{1 / 2}}
$$

The damping ratio in Eq. (39) is calculated as 0.07. The critical damping $\mu_{c}$ is estimated numerically by adjusting the damping parameter $\mu$ in Eq. (32) until the response $\alpha_{1}$ ceases to oscillate. In this way the dimensionless critical damping $\mu_{c}$ is estimated as 10. As a consequence the damping of the assembly is estimated as $\mu=0.7\left(\mu^{*}=9.8 \mathrm{~N} \mathrm{~s} / \mathrm{m}\right)$. It is noted that the concept of critical damping and the logarithmic decrement approach is for a linear oscillator. We assume that the similar concept can be extended to a nonlinear system with a linear viscous damping such as Eq. (32). Although this estimate may appear somewhat engineeringoriented, it is believed that the damping factor in our experimental setup is about this order. Whether this approach is satisfactory is examined by experimental results. The dashed lines in Figs. 8(a) and $8(b)$ are the deflection history from numerically integrating Eq. (32). The short rise time from impact to the peak speed as explained in Fig. 7 is ignored. It is noted that the actual response as measured in the experiment may contain multiple-mode components, while the numerical simulation contains only a single mode $\alpha_{1}$ because of the initial conditions (34). The actual multimode response may result from imperfect initial shape of the arch. The good agreement between the experiment and the numerical simulation as demonstrated in Figs. $8(a)$ and $8(b)$ confirms that the one-mode solution $\alpha_{1}(t) \sin x$ is indeed the dominant component.

\section{Critical Speed Measurements}

Figure 9 shows the results from a series of experiments on the critical initial speed. Figure $9(a)$ shows the relation between the critical initial speed $v_{\mathrm{cr}}^{*}$ and the wall movement $a^{*}$. The fixed parameters of the assembly is $h^{*}=3.46 \mathrm{~cm}(h=80), \quad k^{*}$ $=206 \mathrm{~N} / \mathrm{cm}(k=0.0034)$. The symbol $\times$ represents the measured critical initial speed. In the experiment we increase the striking power of the hammer incrementally until snap-through occurs. The increment in the corresponding initial speed change is about $0.0125 \mathrm{~m} / \mathrm{s}$, which may be considered as the accuracy of the critical speed measurement. Each experiment is repeated three times. Therefore, there are three $\times$ clustered for each specified $a^{*}$. The solid line represents the theoretical predictions. The two black dots represent the wall movement corresponding to $a_{1}$ and $a_{2}$. In the range $a_{2}<a<a_{1}$ the theoretical critical initial speed is given by Eq. (35). In the range $a<a_{2}$ the theoretical critical initial speed is given by Eq. (36). In the range $a>a_{1}$ no snap-through is possible because there exist only one stable equilibrium position $P_{0}$. It is noted that the wall movement $a$ in the experiment ranges from negative to positive values. It is observed that the critical initial speed decreases as the wall movement $a$ increases until the point $a=a_{1}$.

Figure $9(b)$ shows the critical initial speed as a function of the supporting spring constant $k^{*}$. The fixed parameters of the assembly is $h^{*}=3.46 \mathrm{~cm}(h=80), a^{*}=0$. The two points signified by (a)

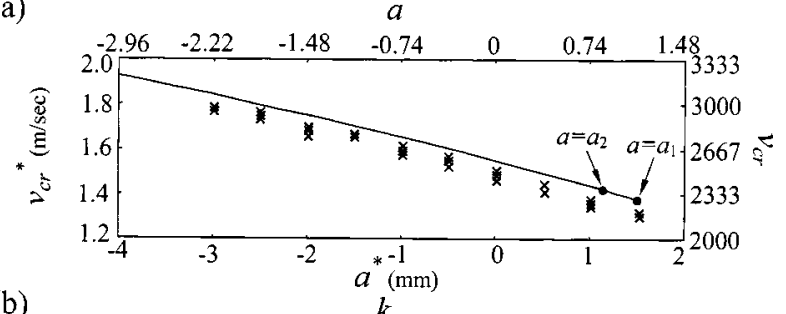

(b)

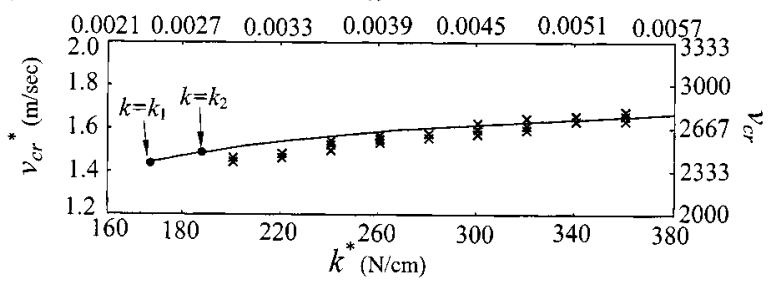

(c)

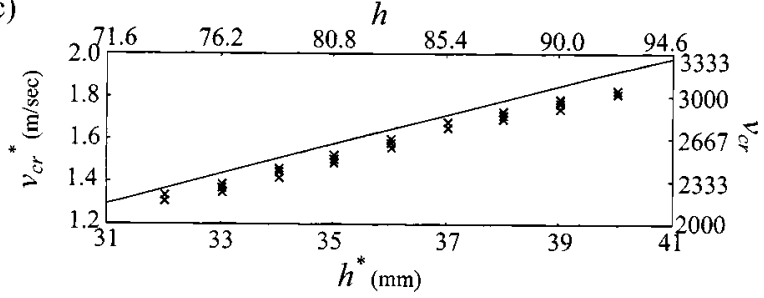

Fig. 9 Critical initial speed $v_{\mathrm{cr}}^{*}$ as a function of (a) wall movement $a^{*},(b)$ supporting spring constant $k^{*}$, (c) initial height $h^{*}$ of the arch. SymbolXrepresents the measured data, while the solid lines are the theoretical predictions.

black dots are $k_{1}=16 / h^{2}$ and $k_{2}=18 / h^{2}$. In the range $k_{1}<k<k_{2}$ the theoretical critical initial speed is given by Eq. (37). In the range $k<k_{2}$ the theoretical critical initial speed is given by Eq. (38). It is observed that the critical initial speed increases as the spring constant $k^{*}$ increases. Figure $9(c)$ shows the critical initial speed as a function of the initial height $h^{*}$ of the arch. The fixed parameters of the assembly is $k^{*}=208 \mathrm{~N} / \mathrm{cm}(k=0.0035), a^{*}$ $=0$. The two points corresponding to $a_{1}$ and $a_{2}$ are outside the range of this figure. The critical initial speed increases as the initial height of the arch increases. Generally speaking, the experimental results of the critical initial speeds are satisfactory compared to the theoretical predictions.

\section{Conclusions}

In this paper we consider a shallow arch with initial height $h$. One end of the arch is attached to a mass $m$ and supported by a spring with constant $k$. After the supporting wall is moved quasistatically a distance $a$ to preload the arch-mass-spring assembly, the attached mass is subjected to impact and attains initial speed $v_{i}$. We are interested in the conditions under which the arch will snap to the other side dynamically. Some results can be summarized in the following.

(1) There are at most two stable equilibrium configurations for any given combination of $a, h$ and $k$. One of them is $P_{0}$, which is always stable. The other is $P_{1}^{-}$, which is stable only in certain range of $a$ and $k h^{2}$.

(2) When $h \leqslant \overline{h_{2}}$ and $a_{2} \leqslant a<a_{1}$, the energy barrier preventing the arch from snapping from $P_{0}$ to $P_{1}^{-}$is the strain energy of $P_{1}^{+}$. In the case when $a<a_{2}$ the energy barrier is the strain energy of $P_{12}^{ \pm}$.

(3) The assembly is safe from dynamic snap-through as long as the initial speed of the attached mass from the impact is smaller than a critical speed. This critical initial speed can be predicted analytically and confirmed by experiments. 
(4) Generally speaking the critical initial speed decreases as $a$ increases, while it increases as $k$ and $h$ increase.

\section{Acknowledgment}

The results presented here were obtained in the course of research supported by a grant from the National Science Council of the Republic of China.

\section{References}

[1] Timoshenko, S. P., 1935, "Buckling of Flat Curved Bars and Slightly Curved Plates," ASME J. Appl. Mech., 2, pp. 17-20.

[2] Fung, Y. C., and Kaplan, A., 1952, "Buckling of Low Arches or Curved Beams of Small Curvature," NACA Technical Note 2840.

[3] Gjelsvik, A., and Bonder, S. R., 1962, "The Energy Criterion and Snap Buckling of Arches," J. Eng. Mech. Div., 88, pp. 87-134.

[4] Franciosi, V., Augusti, G., and Sparacio, R., 1964, "Collapse of Arches Under Repeated Loading," ASCE J. Struct. Div., 90, pp. 165-201.

[5] Schreyer, H. L., and Masur, E. F., 1966, "Buckling of Shallow Arches," J. Eng. Mech. Div., 92, pp. 1-19.

[6] Lee, H. N., and Murphy, L. M., 1968, "Inelastic Buckling of Shallow Arches," J. Eng. Mech. Div., 94, pp. 225-239.

[7] Simitses, G. J., 1973, "Snapping of Low Pinned Arches on an Elastic Foundation,” ASME J. Appl. Mech., 40, pp. 741-744.

[8] Roorda, J., 1965, "Stability of Structures With Small Imperfections," J. Eng. Mech. Div., 91, pp. 87-106.

[9] Simitses, G. J., 1990, Dynamic Stability of Suddenly Loaded Structures, Springer, New York.

[10] Hoff, N. J., and Bruce, V. G., 1954, "Dynamic Analysis of the Buckling of Laterally Loaded Flat Arches," J. Math. Phys., 32, pp. 276-288.

[11] Hsu, C. S., 1967, "The Effects of Various Parameters on the Dynamic Stability of a Shallow Arch," ASME J. Appl. Mech., 34, pp. 349-358.

[12] Hsu, C. S., 1968, "Stability of Shallow Arches Against Snap-Through Under Timewise Step Loads," ASME J. Appl. Mech., 35, pp. 31-39.

[13] Hsu, C. S., Kuo, C. T., and Lee, S. S., 1968, "On the Final States of Shallow
Arches on Elastic Foundations Subjected to Dynamical Loads," ASME J. Appl. Mech., 35, pp. 713-723.

[14] Xu, J.-X., Huang, H., Zhang, P.-Z., and Zhou, J.-Q., 2002, "Dynamic Stability of Shallow Arch With Elastic Supports-Application in the Dynamic Stability Analysis of Inner Winding of Transformer During Short Circuit," Int. J. NonLinear Mech., 37, pp. 909-920.

[15] Humphreys, J. S., 1966, "On Dynamic Snap Buckling of Shallow Arches," AIAA J., 4, pp. 878-886.

[16] Lock, M. H., 1966, "The Snapping of a Shallow Sinusoidal Arch Under a Step Pressure Load," AIAA J., 4, pp. 1249-1256.

[17] Huang, N. N., and Nachbar, W., 1968, "Dynamic Snap-Through of Imperfect Viscoelastic Shallow Arches," ASME J. Appl. Mech., 35, pp. 289-296.

[18] Ariaratnam, S. T., and Sankar, T. S., 1968, "Dynamic Snap-Through of Shallow Arches Under Stochastic Loads," AIAA J., 6, pp. 798-802.

[19] Fulton, R. E., and Barton, F. W., 1971, "Dynamic Buckling of Shallow Arches," J. Eng. Mech. Div., 97, pp. 865-877.

[20] Sundararajan, V., and Kumani, D. S., 1972, "Dynamic Snap-Buckling of Shallow Arches Under Inclined Loads,” AIAA J., 10, pp. 1090-1091.

[21] Lo, D. L. C., and Masur, E. F., 1976, "Dynamic Buckling of Shallow Arches," J. Eng. Mech. Div., 102, pp. 901-917.

[22] Johnson, E. R., and Mclvor, I. K., 1978, "The Effect of Spatial Distribution on Dynamic Snap-Through,” ASME J. Appl. Mech., 45, pp. 612-618.

[23] Johnson, E. R., 1980, "The Effect of Damping on Dynamic Snap-Through," ASME J. Appl. Mech., 47, pp. 601-606.

[24] Huang, K. Y., and Plaut, R. H., 1982, "Snap-Through of a Shallow Arch Under Pulsating Load," Stability in the Mechanics of Continua, F. H. Schroeder, ed., Springer, Berlin, pp. 215-223.

[25] Gregory, Jr., W. E., and Plaut, R. H., 1982, "Dynamic Stability Boundaries for Shallow Arches," J. Eng. Mech. Div., 108, pp. 1036-1050.

[26] Donaldson, M. T., and Plaut, R. H., 1983, "Dynamic Stability Boundaries for a Sinusoidal Arch Under Pulse Loads," AIAA J., 21, pp. 469-471.

[27] Liao, C.-Y., 2003, "Experiment and Analysis of a Flexibly Supported Arch Under Impact at the End," Master thesis, Department of Mechanical Engineering, National Taiwan University, Taipei, Taiwan.

[28] Rao, S. S., 1995, Mechanical Vibrations, 3rd ed., Addision-Wesley, Reading, MA. 